\title{
Hallazgo incidental de hiperostosis frontal interna o síndrome de Morgagni asociado a meningiomas
}

\section{Incidental finding of frontal internal hyperostosis or Morgagni syndrome associated with meningiomas}

\section{Resumen}

La hiperostosis frontal interna consiste en un engrosamiento de la tabla interna del hueso frontal. Normalmente se encuentra en mujeres post menopáusicas. Presentamos un caso de hallazgo autópsico incidental de hiperostosis frontal interna o síndrome de Morgagni asociado a meningiomas en una mujer postmenopáusica.

Palabras clave: Cráneo. Hiperostosis. Patología forense. Antropología forense.

Abstract

Hyperostosis frontalis interna is the accretion of bone on the inner table of the frontal bone. It is commonly found in post-menopausic women. We present an incidental finding of frontal internal hyperostosis or Morgagni syndrome associated with meningiomas in a post-menopausic woman.

Key words: Skull. Hyperostosis. Forensic pathology. Forensic anthropology.

\section{Introducción}

La etiología de la hiperostosis frontal interna es desconocida pero se considera asociada a los cambios hormonales menopáusicos. Puede ser un hallazgo incidental de autopsia en la que se aprecia un engrosamiento de la tabla interna frontal y en algunas ocasiones, como el caso que presentamos, puede estar asociado con meningiomas.

El interés médico-forense de la hiperostosis frontal interna así como de los meningiomas calcificados radica en que su conocimiento puede ayudar en algunos casos a establecer la causa de muerte, entender y/o resolver denuncias por mala praxis, además de ser un hallazgo que puede tener valor identificativo tanto en cadáveres recientes como en estudios antropológicos.

\section{Presentación del caso}

Al servicio de patología forense llegó el caso de una mujer de 66 años con antecedentes de hipertensión arterial, fibromialgia y miastenia gravis diagnosticada 20 años antes. Seguía tratamiento crónico antihipertensivo con amlodipino (10 mg/24 h), furosemida (40 mg/24 h) y losartán postásico (50 mg/24 h), ansiolítico - antidepresivo con duloxetina (30 mg/24 h) e hidorcloruro de amytriptilina $(10 \mathrm{mg} / 24 \mathrm{~h})$, tratamiento para la osteoporosis con calcifediol (800 mg/24 h) y protección gástrica con omeprazol (20 mg/24 h). Una semana antes del fallecimiento consultó en urgencias por cuadro catarral que se trató con ciprofloxacino y carbocisteína con mejoría del cuadro respiratorio. Dos días antes de morir consultó nuevamente en el servicio de urgencias ya que refería un cuadro de debilidad general que se atribuyó al tratamiento antibiótico y se indicó la suspensión del mismo. No constaba en su historial clínico síntomas de cefalea y/o otros síntomas de focalidad neurológica.

La paciente murió de forma súbita presenciada y su marido refirió disnea como el único síntoma previo a la muerte.
M. Subirana Domènech

M. Ortega Sánchez

JI. Galtés Vicente

J. Castellà García

Médicos Forenses. Institut de Medicina Legal de Catalunya

(IMLC).

Servei de Patologia

Forense.

Subdirecció de Barcelona

Correspondencia: M. Subirana i Domènech Institut de Medicina Legal de Catalunya

Servei de Patologia Forense Edifici G, 5 a planta

Gran Vía, 111

Barcelona 08014

E-mail:

25402msd@comb.cat

Fecha de recepción:

12.MAR.2012

Fecha de aceptación:

7.MAY.2012 
Durante la autopsia los hallazgos más destacables fueron los siguientes:

Talla de $149 \mathrm{~cm}$, peso de $80 \mathrm{Kg}$ e Índice de Masa Corporal de $36 \mathrm{Kg} / \mathrm{m}^{2}$, que se correspondía con una obesidad tipo II. Otros hallazgos destacables del examen externo fueron hirsutismo, cicatriz de toracotomía media como consecuencia de timectomía para el tratamiento de la miastenia gravis y edema de extremidades inferiores. Como signos externos de causa violenta se encontró un hematoma glúteo derecho reciente compatible con la caída que sufrió durante el período perimortal y con la posición sentada en el suelo en la que se encontró durante el acto de levantamiento del cadáver.

Los hallazgos más relevantes del examen interno fueron hemopericardio coagulado de 200 g, el corazón pesaba $400 \mathrm{~g}$ (peso esperado $320 \mathrm{~g}$ ) y apreciamos una rotura de $0,5 \mathrm{~cm}$ en la parte anterior media de ventrículo izquierdo por infarto transmural secundario a ateromatosis coronaria de los tres vasos, más marcada en tercio medio de descendente anterior donde obstruía la luz vascular en más de un $75 \%$.

En el cráneo destacó la presencia de hiperostosis frontal interna (Fig. 1), menos marcada en la zona parietal. El grosor del díploe no afectado era de 8 $\mathrm{mm}$ mientras que en las zonas de hiperostosis los grosores eran de entre $1,5 \mathrm{~mm}$ a 2,3 mm. Además se encontraron múltiples meningiomas calcificados siguiendo el seno longitudinal superior, la mayoría de ellos de forma nodular y algunos de ellos de menor tamaño, espiculados y con forma de placa (Fig. 2). A nivel cerebral se apreció una ligera depresión de la superficie de ambos lóbulos frontales.

El resto de hallazgos de autopsia no fueron significativos y los resultados del estudio analíticos toxicológicos fueron negativos. El estudio histopatológico fue concordante con los datos macroscópicos de hiperostosis frontal interna y los meningiomas eran psamomatosos calcificados.

\section{Discusión}

La interpretación de los hallazgos de autopsia, cuando los contrastamos con el historial clínico de la paciente, orientaron la posibilidad de que el cuadro catarral diagnosticado y tratado con ciprofloxacino y carbocisteína podía ser concomitante o haber enmascarado una isquemia miocárdica que terminó con rotura del infarto transmural.

Además tenemos dos entidades como hallazgos incidentales de autopsia: la hiperostosis frontal interna y la presencia de meningiomas sobre los que centraremos la discusión.

\section{Introducción histórica}

La hiperostosis frontal interna fue descrita en el siglo XVIII por Morgagni ${ }^{1}$ tras apreciar engrosamiento frontal interno en la autopsia de una mujer con obesidad e hirsutismo.

El término meningioma fue introducido por Cushing en 1922 en relación con la histogénesis de estos tumores y las cubiertas meníngeas pero años antes, en el 1904, Winker describió un meningioma de localización extradural².

\section{Criterios diagnósticos}

La hiperostosis frontal interna consiste en un engrosamiento de la tabla interna del hueso frontal. En medicina forense se detecta durante la autopsia craneal o bien durante el estudio radiológico-antropológico.

En el estudio radiográfico presenta una mayor densidad del hueso con engrosamiento a nivel frontal que se compara con la forma de una mariposa, generalmente asociada a un trastorno de la arquitectura tisular. Se describe en un $5-15 \%$ de la población general y en mujeres menopáusicas entre el $40-60 \%{ }^{3}$, más raramente en individuos jóvenes $^{4} \mathrm{o}$ en varones con testículos atróficos ${ }^{5}$. No hemos encontrado en nuestra búsqueda bibliográfica series de detección en autopsia.

La predilección por la zona frontal puede considerarse relacionada con el aporte sanguíneo ${ }^{5}$.

Encontramos distintas denominaciones según la asociación de la hiperostosis con clínica:

- Síndrome de Stewart Morel: hiperostosis frontal interna, obesidad y alteraciones mentales ${ }^{6}$.

- Síndrome de Troell-Junet: hiperostosis frontal interna, acromegalia, gota y diabetes mellitus.

Los menigiomas surgen de las células aracnoideas de las leptomeninges y son tumores de crecimiento lento. Constituyen entre el $14-18 \%$ de las neoplasias intracraneales con una incidencia de 2-3 casos por cada 100.000 habitantes con mayor frecuencia en las mujeres de mediana edad ${ }^{7}$ y desde el punto de vista asistencial, la posibilidad de que un meningioma intraóseo simule una metástasis siempre debe tenerse en cuenta².

\section{Etiología}

La etiología de la hiperostosis frontal interna es desconocida pero se considera asociada a los cambios hormonales menopáusicos y en varones ancianos con hipogonadismo $^{8}$. Se postula también una base genética ${ }^{9-11}$. 
En cuanto a los meningiomas algunos autores refieren que se forman como consecuencia del atrapamiento de las células aracnoideas en las suturas intracraneales ${ }^{12}$, otros autores consideran un origen embrionario y en algunos casos se ha documentado un origen traumático ${ }^{13}$ y conlleva problemas diagnósticos en aquellos casos en los que subyace una neoplasia de base ${ }^{14}$.

Por tanto, vemos que, como en el caso presentado, se asocia a mujeres a partir de la edad media, pero en la autopsia que realizamos no existía neoplasia de origen extracraneal ni la evidencia en el historial clínico examinado de antecedentes traumáticos. Los hallazgos de hirsutismo y obesidad serían concordantes con la descripción de síndrome de Morgagni.

\section{Diagnóstico diferencial}

Como causas de hiperostosis craneal encontramos el síndrome de Klippel-Trenaunay- Weber, el síndrome de Frölich, la enfermedad de Paget, la leontiasis ósea, el meningioma, el osteoma endosteal , los osteofitos asociados al embarazo, el síndrome de Morgagni y la displasia fibrosa ${ }^{15,16}$. Estas entidades deben centrar el diagnóstico diferencial con la hiperostosis frontal interna. Para ello pueden ser útiles los siguientes criterios:

- El síndrome de Klippel-Trenaunay- Weber, síndrome de Frölich y la enfermedad de Paget se caracterizan por afectar la base del cráneo. La enfermedad de Paget así como la acromegalia y displasia fibrosa afectan el díploe y ectocráneo.

- La leontiasis ósea (o facies leonina) se ven afectados tanto los huesos craneales como los faciales en fases avanzadas de la lepra.

- Los meningiomas así como los osteomas inducen a calcificaciones localizadas.

- Los osteofitos asociados al embarazo se localizan en la bóveda craneal, más frecuentes en el ectocráneo y se encuentran preferentemente en áreas de senos venosos.

- La acromegalia, como trastorno endocrino secundario a la secreción excesiva de hormona del crecimiento, afecta a los huesos largos con un engrosamiento en las zonas de cartílago de crecimiento ya fusionadas ${ }^{15}$.

- Finalmente, el síndrome de Morgagni requiere la presencia de obesidad y virilismo en asociación a hiperostosis frontal interna.

Desde una visión antropológica, en la mayoría de las ocasiones no se pueden contrastar los hallazgos con la historia clínica y/o exploraciones complementarias. Ha sido ampliamente estudiado y llama la atención la descripción de 11 hallazgos de hiperostosis frontal interna en una serie 37 esqueletos ${ }^{17}$ y el estudio de un esqueleto con cráneo con características masculinas mientras que el resto del mismo era de características femeninas ${ }^{18}$. Hershkovitz realizó un exhaustivo estudio antropológico y clasificó la hiperostosis frontal interna en cuatro tipos de la $A$ a $D$ (de menor a mayor extensión). En los casos avanzados, C y D aunque también se apreciaba en menores de 40 años era más frecuente a partir de los 60 y la frecuencia del tipo D no aumentaba a partir de los 60 años $^{5}$.

La detección de meningiomas en casos de estudios antropológicos no es frecuente ya que su observación dependerá de que éstos estén calcificados. En el caso que hemos descrito, si se hubiera estudiado al cabo de unos años y siempre desde una perspectiva antropológica, hubiéramos apreciado la persistencia de la hiperostosis frontal interna así como de la persistencia de meningiomas ya que éstos estaban calcificados y hubieran resistido el paso del tiempo.

\section{Síntomas asociados}

Como responsable de los síntomas de la hiperostosis frontal interna en la revisión bibliográfica apreciamos que se ha asociado a cefalea migrañosa, vértigo, hirsutismo, trastornos menstruales, galactorrea, obesidad, depresión, irritabilidad, fatiga, hemiplejias transitorias, trastornos auditivos, parálisis de los nervios craneales, debilidad muscular, disfunción vestibular y epilepsia ${ }^{19}$. Actualmente se considera la hiperostosis frontal interna como una condición independiente más que la forma generalizada de un síndrome. La presentación como metástasis de duramadre con hiperostosis de calota es infrecuente $^{20,21}$ y las neoplasias más frecuentemente asociadas a esta entidad son las de mama y próstata ${ }^{22-25}$. En el cáncer de mama es más frecuente en el lobular infiltrante, mientras que en el ductal infiltrante las metástasis son con mayor frecuencia cerebrales $^{26}$. El adenocarcinoma de localización primaria desconocida es un problema clínico frecuente. Sin embargo, la presentación como metástasis en duramadre con hiperostosis de calota es inusual. Entre las neoplasias que con mayor frecuencia metastatizan a este nivel destacan las de mama y próstata. En cuanto al cáncer de mama, suele asociarse al subtipo lobular infiltrante que tiende a metastatizar a médula ósea y desde ahí infiltra meninges, a diferencia del ductal infiltrante con predisposición a producir metástasis en parénquima cerebral. Entre un $80-85 \%$ de los casos no se consigue localizar el tumor primario ${ }^{25}$.

Las manifestaciones clínicas de los meningiomas varían según la localización específica, pero de 
forma general ocasionan cefalea, crisis convulsivas en su mayoría focales, hemiparesia, trastornos oculares, alteraciones de las funciones psíquicas superiores e hipertensión endocraneana ${ }^{27,28}$.

Nos podemos plantear el por qué en los casos de meningioma se produce una invasión ósea o inducción de hiperostosis como en el caso objeto de estudio. Como hipótesis han surgido explicaciones como la alteración vascular del hueso como consecuencia del tumor o una formación osteoblástica del tejido óseo originada por factores secretores (PDGF, IGF-1, IGF-2, TGF beta 1 y 2). Heick et al sugirieron que los hallazgos de hiperostosis podían ser consecuencia de la actividad enzimática de la fosfatasa alcalina que tendría propiedades de osificación en los meningiomas con hiperostosis ${ }^{29,30}$. Desconocemos la

\section{Bibliografía}

1. Moore S:. Hyperostosis Cranii. Springfield Illinois: Charles C Thomas, 1955. pp 3-195.

2. Yener U, Bayrakli F, Vardereli E, Sav A, Peker S. Intradiploic Meningioma. Mimicking Calvarial Metastasis: Case Report. Turkish Neurosurgery 2009; 19:3:297-301.

3. Querol MR, Aguirre JJ, Gahete C. Neuroimaging signs in a case of frontal internal hyperostosis. Neurologia 2002;17(8):437.

4. Hrycek A, Błoch B, Szołtysik I. Morgagni-StewartMorel syndrome in a young man. Wiad Lek 1989;42(19-21):1060-3.

5. Hershkovitz I, Greenwald C, Rothschild BM, Latimer B, Dutour O, Jellema LM, Wish-Baratz S. Hyperostosis frontalis interna: an anthropological perspective. Am J Phys Anthropol 1999;109(3):303-25.

6. Hrycek A, Błoch B, Szołtysik I. Morgagni-StewartMorel syndrome in a young man. Wiad Lek 1989;42(19-21):1060-3.

7. Baek JU, Cho YD, Yoo JC. An osteolytic meningioma en plaque of the sphenoid ridge. J Korean Neurosurg Soc 2008;43(1):34-6.

8. Yamakawa K, Mizutani K, Takahashi M, Matsui M, Mezaki T. Hyperostosis frontalis interna associated with hypogonadism in an elderly man. Age Ageing 2006;35(2):202-3.

9. Koller MF, Papassotiropoulos A, Henke K, Behrends B, Noda S, Kratzer A, Hock C, Hofmann M. Evidence of a genetic basis of Morgagni-Stewart-Morel syndrome. A case report of identical twins. Neurodegener Dis 2005;2(2):56-60. incidencia que tuvo en el caso presentado el tratamiento para la osteoporosis con calcifediol (25-hidroxicolecalciferol o 25-hidroxivitamina D).

Por tanto, el caso presentado presenta la tríada del síndrome de Morgagni: hiperostosis frontal interna, obesidad e hirsutismo en una mujer postmenopáusica como hallazgos incidentales de una autopsia.

\section{Agradecimientos}

Nuestro más sincero agradecimiento a los técnicos especialistas en patología forense del IMLC BarceIona y a Celia Rudilla del servicio de biblioteca.
10. Klein D, Rosatti P, Mach RS, Ferrier PE, Rauch S Morgagni-Morel syndrome an independent clinical and genetic entity, demonstrated in a family study covering 4 generations]. Schweiz Arch Neurol Neurochir Psychiatr 1973;112(2):239-50.

11. Glab H, Szostek K, Kaczanowski K. Hyperostosis frontalis interna, a genetic disease?: Two medieval cases from Southern Poland. Homo 2006;57(1):19-27.

12. De Geeter F, Delanote J, Goethals L, Vandelanotte $\mathrm{S}$, Vannerem L. Scintigraphic imaging of intraosseous sphenoid meningioma. Clin Nucl Med 2009;34(8):535-6.

13. Crawford TS, Kleinschmidt-DeMasters BK, Lillehei KO.Primary intraosseous meningioma. Case report. J Neurosurg 1995;83(5):912-5.

14. Cotrina Monroy AP, López López A, Ruiz Solis S, Gómez Embuena A. Incidental finding of a meningioma-en-plaque in a patient with prostate adenocarcinoma. Rev Esp Med Nucl 2010 ;29(5): 254-7.

15. Talarico EF Jr, Prather AD, Hardt KD. A case of extensive hyperostosis frontalis interna in an 87-year-old female human cadaver. Clin Anat. 2008;21(3):259-68.

16. She R, Szakacs J. Hyperostosis frontalis interna: case report and review of literature. Ann Clin Lab Sci 2004;34(2):206-8.

17. Mulhern DM, Wilczak CA, Dudar JC. Brief communication: unusual finding at Pueblo Bonito: multiple cases of hyperostosis frontalis interna. Am J Phys Anthropol 2006;130(4):480-4.

18. Martinez Gómez AL, Peral Pacheco D, Vallejo JR, Sánchez Sánchez J A. Un posible caso de Síndrome de Morgagni-Stewart-Morel en un esqueleto de la 
colección de restos óseos de la Escuela de Medicina Legal de Madrid. Revista de la Escuela de Medicina Legal 2007;5:40-54.

19. Nallegowda M, Singh U, Khanna M, Yadav SL, Choudhary AR, Thakar A. Morgagni Stewart Morel syndrome--additional features. Neurol India 2005;53(1):117-9

20. Mustafa MS, Al-Nuaim L, Inayat-Ur-Rahman N Scalp and cranial bone metastasis of endometrial carcinoma: case report and literatura review. Gynecol Oncol 2001;81:105-9.

21. Smith DB, Howell A, Harris M, Bramwell VH, Sellwood RA. Carcinomatous meningitis associated with infiltrating lobular carcinoma of the breast. Eur J Surg Oncol 1985;11:33-6.

22. Tomlin JM, Alleyne $\mathrm{CH}$. Transdural metastasis from adenocarcinoma of the prostate mimicking subdural hematoma: case report. Surg Neurol 2002;58(5):329-31

23. Varlet, et al. Secondary subdural hematoma in dural metastasis of prostatic adenocarcinoma. Rev Neurol (Paris) 2001;157:1543-5.

24. Scarrow AM, Rajendran PR, Marion D. Metastatic prostate adenocarcinoma of the dura mater. $\mathrm{Br} J$ Neurosurg 2000;14:473-4.
25. Suárez Álvarez L, Muelas Gómez N, Todolí Parra JA Sevilla Mantecón T. Calabuig Alborch J. R. Hiperostosis craneal como forma de presentación de un adenocarcinoma metastásico. An Med Interna 2004;21:548-50.

26. Adams RD, Victor M, Ropper AH. En: Principios de Neurología. Mc Graw-Hill Interamericana; 1999; pp 575-9.

27. Guillermo Pardo C, daConceiçao A Fernando, Casares D Jorge A, Cardoso N Oscarlyns, Varela H Ariel. Cirugía de los meningiomas intracraneales en el Servicio de Neurocirugía del Hospital Provincial Docente "Manuel Ascunce Domenech". Rev Chil Neuro-Psiquiatr 2009;47(2):124-31.

28. Jiang XB, Ke C, Han ZA, Lin SH, Mou YG, Luo RZ, Wu SX, Chen ZP. Intraparenchymal papillary meningioma of brainstem: case report and literature review. World J Surg Oncol 2012;10:10.

29. Sanson M, Cornu P.Biology of meningiomas. Acta Neurochir (Wien) 2000;142(5):493-505

30. Pieper DR, Al-Mefty O, Hanada Y, Buechner D. Hyperostosis associated with meningioma of the cranial base: secondary changes or tumor invasion. Neurosurgery 1999;44(4):742-746.

\section{Anexo fotográfico}

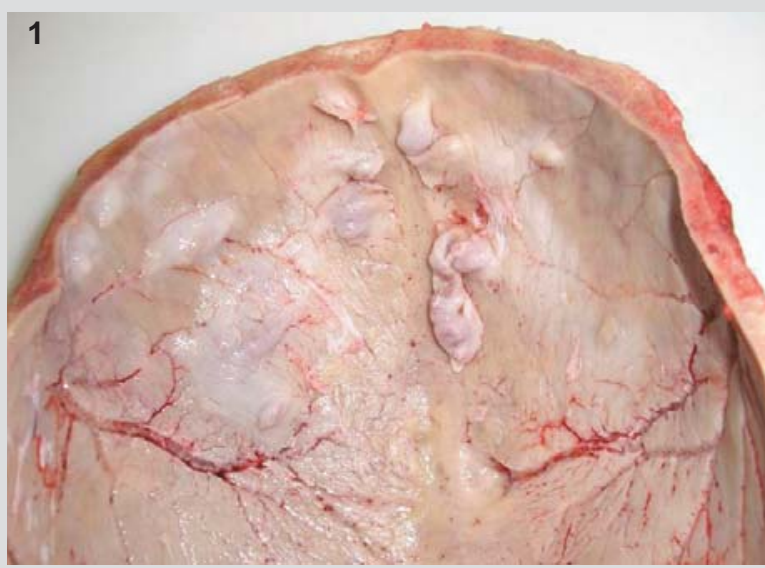

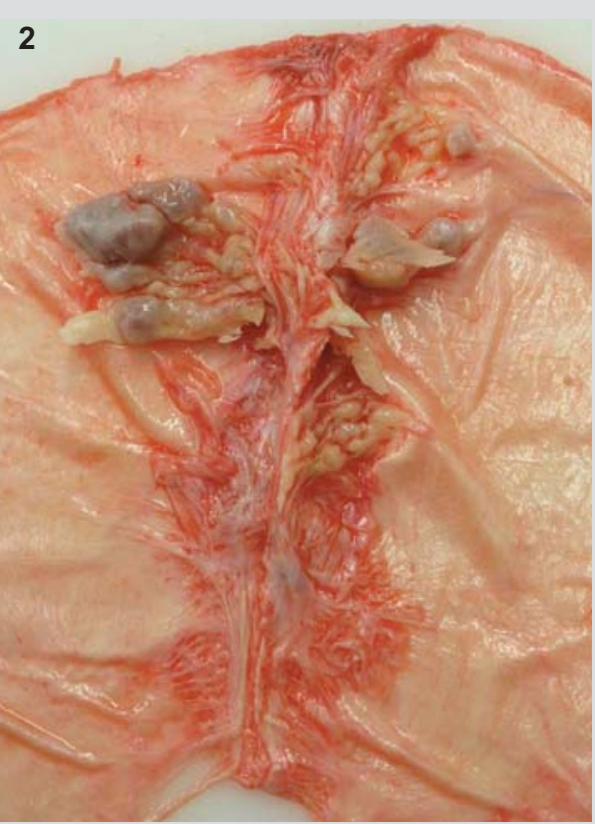

\section{Figura 1.}

Engrosamiento de la tabla interna frontal: hiperostosis frontal interna.

\section{Figura 2.}

Meningiomas calcificados nodulares, algunos de ellos de menor tamaño, espiculados y con forma de placa. 